\title{
Surface Structure and Chemical Reactivity of Electrodeposited Nickel
}

\author{
By Koji Hashimoto*
}

\begin{abstract}
Structure and distribution of chemically reactive areas have been studied on electrodeposited polycrystalline nickel having (211), (210) and (110) preferred orientations by means of electron microscopy. These characteristic preferred orientations occur in nickel specimens more than a few $\mu$ in thickness electrodeposited on mechanically polished, copper substrates. Special procedures have been devised in order to directly observe the structures of thin top layers of the thick deposits having the preferred orientations by means of transmission electron microscopy.

A large number of hillocks with the (I11 )twins are formed in an electrodeposited nickel with the (211) orientation. This result was previously obtained by the author by means of reflection electron diffraction and electron microscopy using replicas, and has been confirmed in the present study. It has also been found that twin and grain boundaries in these deposits are chemically reactive and dissolved initially in a corrosive solution. In an electrodeposited nickel with the (210) orientation, twin boundaries and stacking faults are dissolved during immersion in the corrosive solution. In an electrodeposited nickel with the (110) orientation, the twinning is seldom formed, and the deposit shows no areas remarkable in chemical reactivity except grain boundaries. The electrodeposited nickel with the (211) orientation prepared by the present electrodepositing conditions appears to be more chemically reactive than the deposits having other orientations, because a high density of chemically reactive crystal defects is found in the former.
\end{abstract}

(Received. July 10, 1965)

\section{Introduction}

The electrodeposition is often carried out for the purpose of forming a protective coating against corrosive environment. The author(1) investigated the relationship between the crystal structure and the chemical reactivity of nickel electrodeposited on chemically polished single crystals of copper, and concluded from their early dissolution in a corrosive solution that twinning dislocations and twin boundaries were the most chemically reactive areas. The distribution of chemically reactive crystal defects depends upon the surface structure of electrodeposited metals, and therefore the corrosion resistance against corrosive environment is expected to depend upon the structure, too.

It has been $\operatorname{known}^{(2),(3)}$ that the structure of electrodeposited metals is substantially affected by that of the substrates at an early stage of electrodeposi-

* The reseach Institute for Iron, Steel and Other Metals, Tohoku University, Sendai, Japan.

(1) K. Hashimoto: Trans. JIM, 4 (1963), 142.

(2) G.I. Finch and C.H. Sun: Trans. Faraday Soc., 32 (1936), 852.

(3) G.I. Finch and A.C. Williams: Trans. Faraday Soc., 33 (1937), 564. tion, but with increase of the thickness, characteristic preferred orientations independent of the orientation of the substrate appear. In the electrodeposited nickel the preferred orientations in which (100), (110), (210), and/or (211) planes are parallel to the substrate surface occur depending upon the electrodepositing conditions such as the composition of solution, $\mathrm{pH}$, current density, temperature, additives, stirring, etc. (4),(5). The author(6) proposed from the results of observations by electron microscopy using replicas and reflection electron diffraction that there are considerable differences in the twin formation among surface structures of the electrodeposited nickel having the (110), (210) and (211) orientations. Particularly, he pointed out a misinterpretation of experimental results in many of the previous studies $(4),(5),(7),(8)$ by the reflection electron diffraction method which concluded that close-packed hexagonal nickel crystals

(4) B.C. Banerjee and A. Goswami: J. Electrochem. Soc., 106 (1959), 20.

(5) H. Okada, I. Takamura and T. Ishida: Bull. Univ. Osaka Prefecture 8 (1959), 83.

(6) K. Hashimoto: Trans. JM, 6 (1965), 166.

(7) L. Yang: J. Electrochem. Soc., 97 (1950), 241.

(8) A.K.N. Reddy: Acta Cryst., 17 (1964), 443. 
having the (1010) orientation are contained in facecentered cubic nickel deposits with the (211) orientation. It was also made clear that a kinematical double diffraction spot from hillocks twinned on the (111) plane which makes an angle of $90^{\circ}$ with the surface corresponds accidentally to the (1010) spot of the closepacked hexagonal phase with the (10ī0) orientation in reflection electron diffraction patterns.

Concerning the corrosion resistance of electrodeposited nickel against corrosive atmosphere, it is of great interest to study the distribution of chemically reactive areas in the electrodeoposited nickel having remarkably different surface structures. In a previous study ${ }^{(6)}$ by means of reflection electron diffraction and electron microscopy using replicas, it was difficult to detect crystal defects and electronmicroscopically small areas in the electrodeposited nickel which are most liable to corrosive atmosphere. In the present paper, thin films were extracted from the surfaces of thick nickel deposits, and the surface structure was directly observed in detail by transmission electron microscopy. The distribution of the chemically reactive areas and the difference in chemical reactivity among the deposits with different orientations are discussed.

\section{Experimental Procedures}

In an experiment(1) in which a chemically polished copper specimen was used as the substrates, the orientation of the deposits depended upon that of each crystal grain consisting of the substrate and was not reproducible as a whole. As it is necessary for good reproducibility of the orientation to keep the surface condition of the substrates constant, mechanically polished copper disks were used as the cathode in the present case. The anode was an electrolyzed nickel plate. Composition and $\mathrm{pH}$ of electrodepositing solutions are given in Table 1 . Electrodeposition was carried out using the current density of $47 \mathrm{~mA} / \mathrm{cm}^{2}$ at room temperature and the solutions were unstirred.

Table 1 The composition and $\mathrm{pH}$ of electrolytic solutions

\begin{tabular}{l|ll|l|ll}
\hline \multirow{2}{*}{ Composition } & \multicolumn{4}{|c}{ Orientation of the deposits } \\
\cline { 2 - 6 } & \multicolumn{2}{|c|}{$(211)$} & \multicolumn{1}{|c|}{$(210)$} & \multicolumn{2}{|c}{$(110)$} \\
\hline $\mathrm{NiCl}_{2} \cdot 6 \mathrm{H}_{2} \mathrm{O}$ & 1 & $\mathrm{M} / \mathrm{L}$ & $0.18 \mathrm{M} / \mathrm{L}$ & 0 & $\mathrm{M} / \mathrm{L}$ \\
$\mathrm{NiSO}_{4} \cdot 7 \mathrm{H}_{2} \mathrm{O}$ & 0 & & 0.82 & 1 & \\
$\mathrm{H}_{3} \mathrm{BO}_{3}$ & 0.48 & 0.48 & 0.48 \\
$\mathrm{pH}$ & 4 & 4 & 4 \\
\hline
\end{tabular}

Eelectrodeposited crystals having a characteristic preferred orientation were found in the deposits more than a few microns in thickness. In order to directly observe the surface structure of the deposits by transmission electron microscopy, the surface layers of several hundred angstroms must be extracted from the thick deposits several microns thick. Utilizing the property(1) that metals with the same facecentered cubic structure as the substrate metal grow, in general, epitaxially during electrodeposition on the single crystal substrates, the following procedures were adopted to extract a thin surface layer: (1) Nickel was electrodeposited $5 \mu$ in thickness on the copper substrate and the specimen was rinsed by distilled water. (2) Copper was deposited $3000 \AA$ in thickness on the electrodeposited nickel and the specimen was rinsed by distilled water. (3) Nickel was further electrodeposited $400 \AA$ in thickness on these copper deposits under the same condition as that in the procedure (1) and the specimen was rinsed by distilled water. (4) The electrodeposited copper of the middle layer was dissolved, and the electrodeposited nickel of the outermost layer $400 \AA$ thick was isolated and rinsed by distilled water and methyl alcohol. The outermost nickel layer was used for the specimen of transmission electron microscopy. In order to detect chemically reactive areas, the specimens were immersed in a corrosive solution following the procedure (3) and were subsequently subjected to the procedure (4). The electrodepositing solution of copper deposition was an aqueous solution consisting of $250 \mathrm{~g} / \mathrm{L}$ of $\mathrm{CuSO}_{4} \cdot 5 \mathrm{H}_{2} \mathrm{O}$ and $60 \mathrm{~g} / \mathrm{L}$ of $\mathrm{H}_{2} \mathrm{SO}_{4}$. The current density of copper deposition was $30 \mathrm{~mA} / \mathrm{cm}^{2}$. When the thickness of the middle layer of copper deposits was less than $1000 \AA$, the copper layer could not be dissolved, and with the thickness more than $6000 \AA$, the structure of the inner thick nickel deposits was not perfectly inherited by the top thin nickel deposits. The electrodeposited copper of the middle layer was dissolved using a mixed aqueous solution of $500 \mathrm{~g} / \mathrm{L}$ of $\mathrm{Cr}_{2} \mathrm{O}_{3}$ and $50 \mathrm{~g} / \mathrm{L}$ of $\mathrm{H}_{2} \mathrm{SO}_{4}$. It took about one and a half hours to completely dissolve the electrodeposited copper of the middle layer. The electrodeposited nickel was corroded by immersing in a $5 \% \mathrm{FeCl}_{3}-\mathrm{CH}_{3} \mathrm{OH}$ solution.

\section{Results and Discussion}

For convencience sake, the electrodeposited nickel $5 \mu$ thick on mechanically polished copper disks is named the Ist type electrodeposits, and the electrodeposited nickel $400 \AA$ thick on copper deposits $3000 \AA$ thick on the 1st type electrodeposits is called the 2nd type electrodeposits.

\section{Electron diffraction patterns}

Reflection electron diffraction patterns obtained from the surfaces of the 1st and 2nd type electrodeposits with the (211) orientation are shown in Photos. 1 and 2, respectively. The (111) spot indicated by a double arrow in Photo. 2 appears in a direction perpendicular to the shadow edge. This shows the occurrence of crystals having the (111) orientation, with crystals with the same orientation as the inner nickel deposits with the (211) orientation as a result of deposition of the copper middle layer. The extra spot indicated by a single arrow in Photo. 2 is also observed in a way similar to the 1st type electrodeposits as shown in Photo. 1. The reason for the occurrence of the extra spot has already been elucidated by the author ${ }^{(6)}$ as follows: A large number of hillocks twinned on the (i11) plane which makes an angle of $90^{\circ}$ with the (211) orientation plane grow on the surface as shown in Photo. 3. A kinematical 


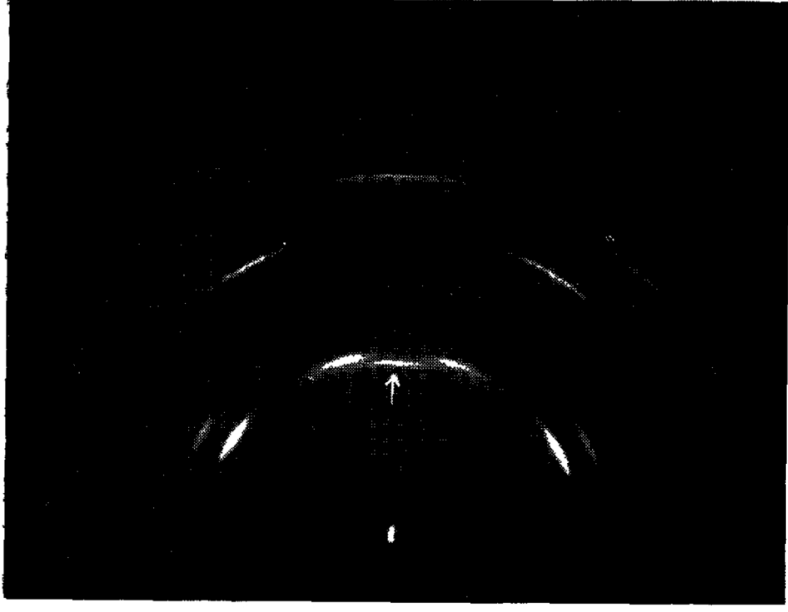

Photo. I Reflection electron diffraction pattern obtained from the lst type electrodeposits with the (211) orientation (6).

An extra spot is indicated by an arrow.

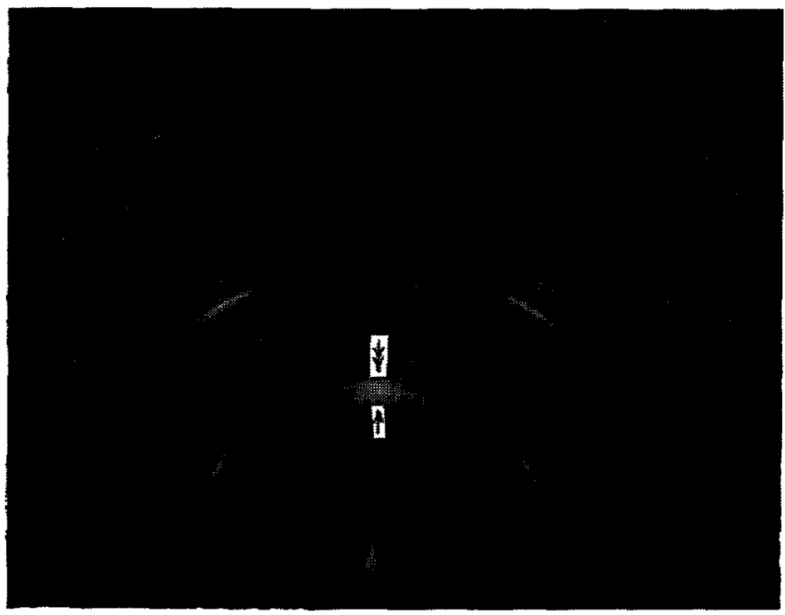

Photo. 2 Reflection electron diffraction pattern obtained from the 2nd type deposits having the (211) orientation. Diffraction spots of crystals with the (111) orientation also appear. Spots indicated by single and double arrows are the extra spot and the (111) spot, respectively.

double diffraction spot arises from the reflection from the $(1 \overline{1} 1)_{\mathrm{M}}$ or $(11 \overline{1})_{\mathrm{M}}$ plane of the crystal $\mathrm{M}$ lying on one side of the twin plane and the $(11 \overline{1}) z_{1}$ or $(1 \overline{1} 1) z_{1}$ plane of the crystal $Z_{1}$ on the other side, and this double diffraction spot corresponds to the $1 / 3(422)$ reflection. The double diffraction spot in Photo. 2 has a diffracted intensity comparable with the (111) spot caused by the primary reflection. It is, therefore, probable that the structure of the inner thick nickel deposits was almost inherited by that of the outermost thin nickel deposits. This is supported by the transmission electron diffraction pattern of the 2 nd type electrodeposits shown in Photo. 4 which indicates a very high diffracted intensity of the (111) ring and an extremely low intensity of the (200) ring.

\section{The surface structure}

\section{(1) Experimental results}

A transmission electron micrograph obtained from the 2nd type electrodeposits with the (211) orientation is shown in Photo. 5. Crystals in which parallel

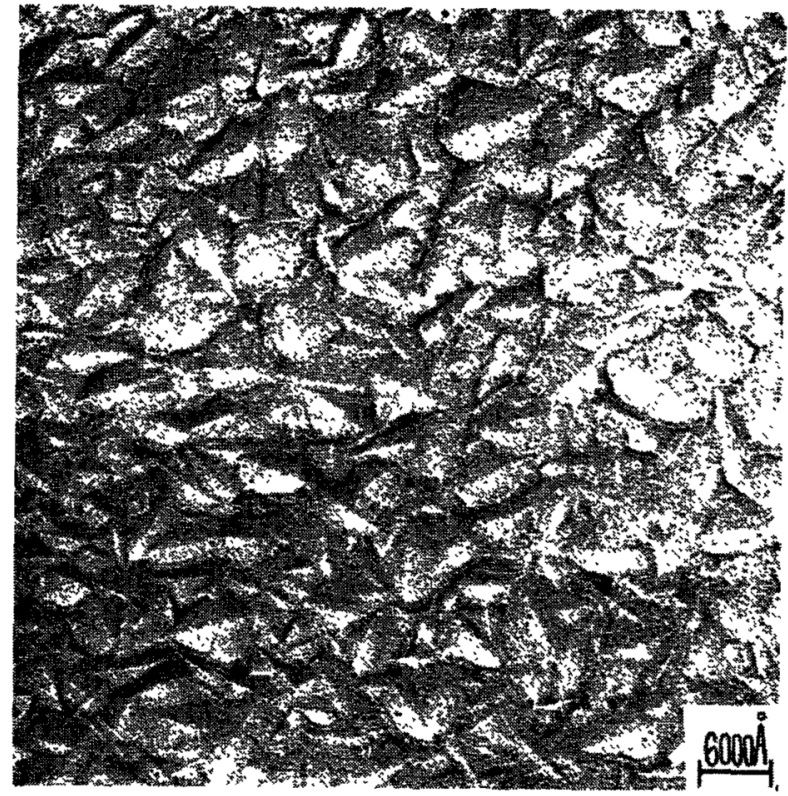

Photo. 3 Electron micrograph obtained from the 1st type electrodeposits with the (211) orientation $^{(6)}$.

An acetyl cellouse carbon replica was used.

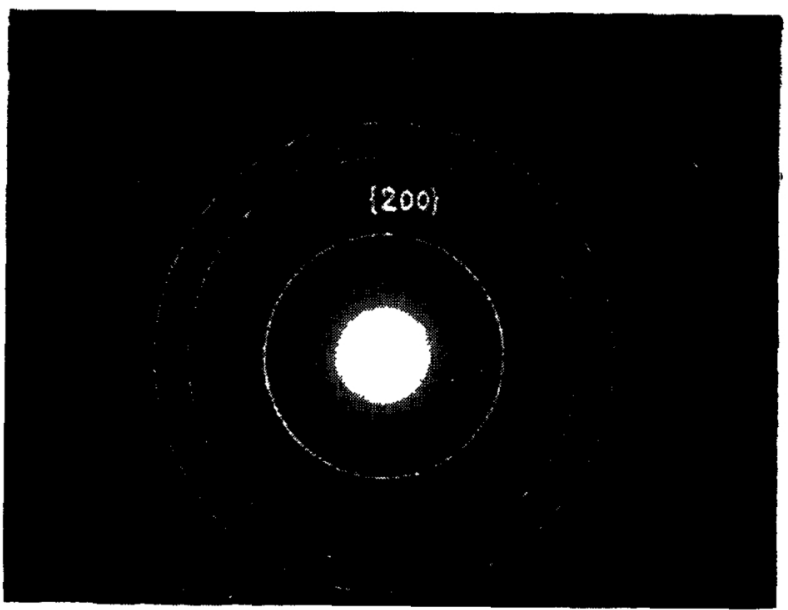

Photo. 4 Transmission electron diffraction pattern obtained from the 2nd type deposits having the (211) orientation

streaks appear lengthwise are observed in large numbers. Such crystals are observed innumerably all over the electron micrograph. The surface of the 1st type electrodeposits is covered by a large number of hillocks as shown in Photo. 3, and the size of the crystals shown in Photo. 5 is comparable with that of the hillocks in Photo. 3. One of these crystals seems, therefore, to correspond to one of the hillocks in Photo. 3. A transmission electron micrograph of a hillock is shown in Photo. 6. Photo. 7 shows an electron diffraction pattern of the rectangular field limited by a diaphragm in Photo. 6. Since the size of the hillock is very small, the beam diffracted by its neighboring crystals necessarily participates in the image formation. In consequence, diffraction spots from the neighboring crystals appear together with those from the hillock of the area selected. Dark field images formed by the diffraction spots indicated by $(1 \overline{1} 1)_{\mathrm{M}}$ and $(002)_{\mathrm{Z}_{1}}$ are shown in Photos. 8-a and 


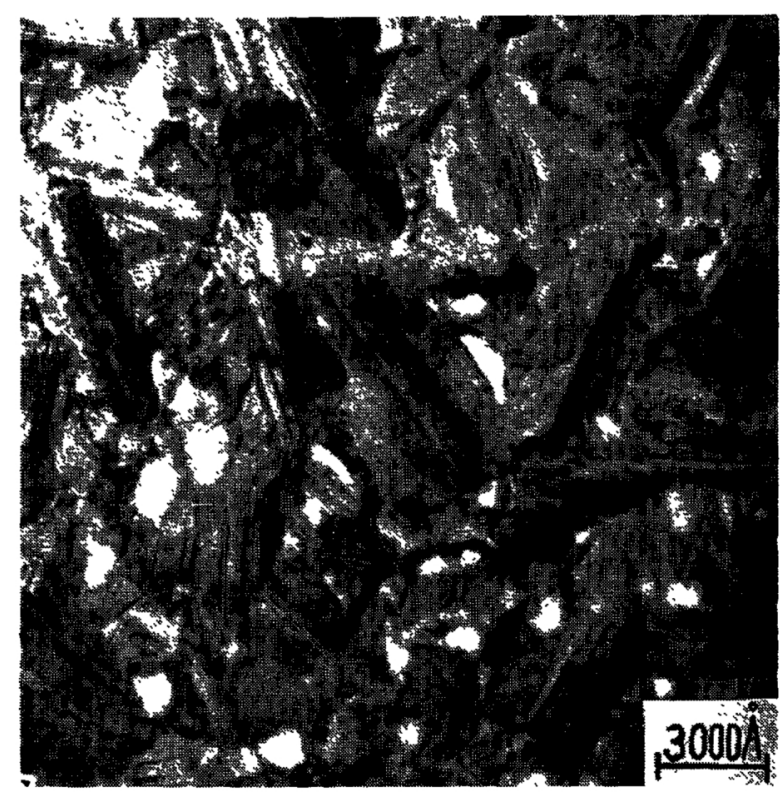

Photo. 5 Transmission electron micrograph of the deposits having the $(211)$ orientation

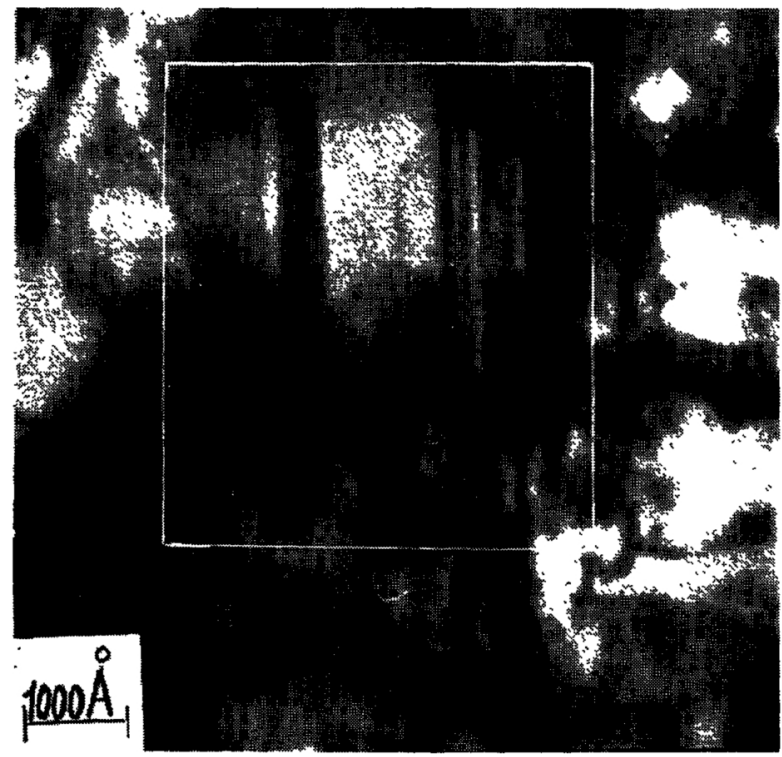

Photo. 6 Bright filed image of a hillock in the electrodeposits with the (211) orientation

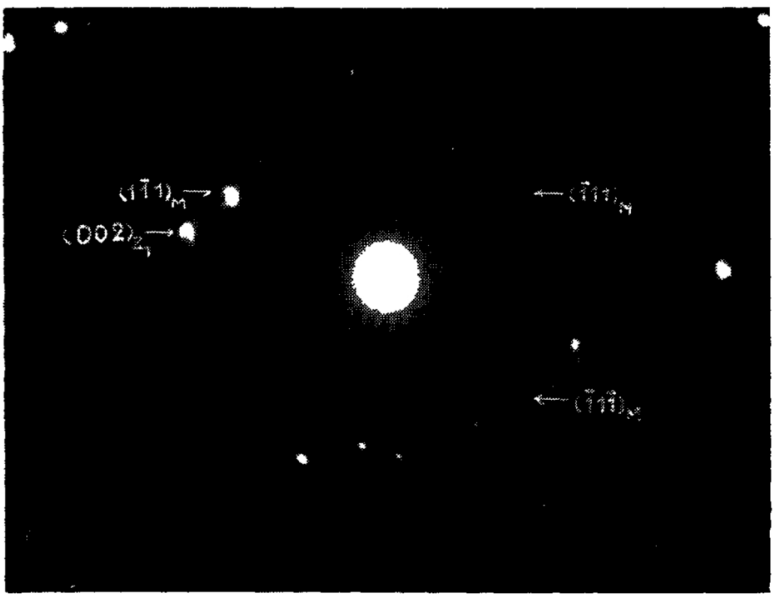

Photo. 7 Selected area electron diffraction pattern of the rectangular area in Photo. 6.

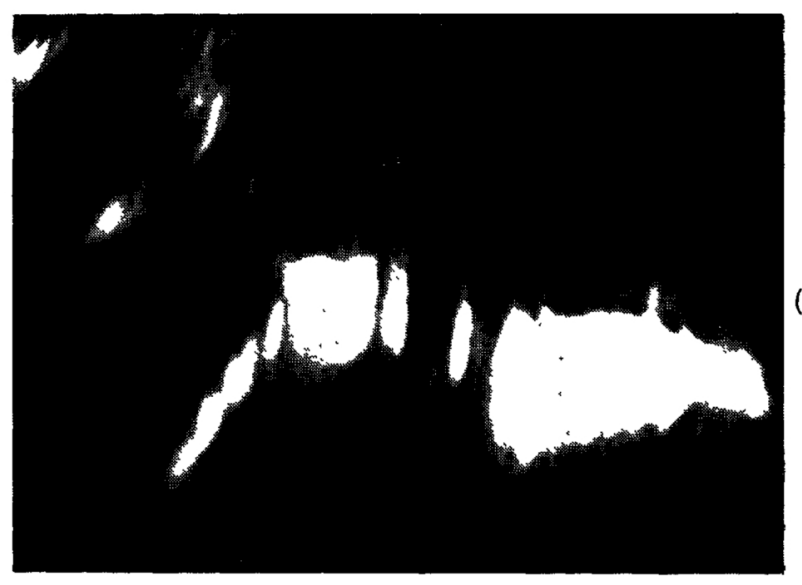

(a)

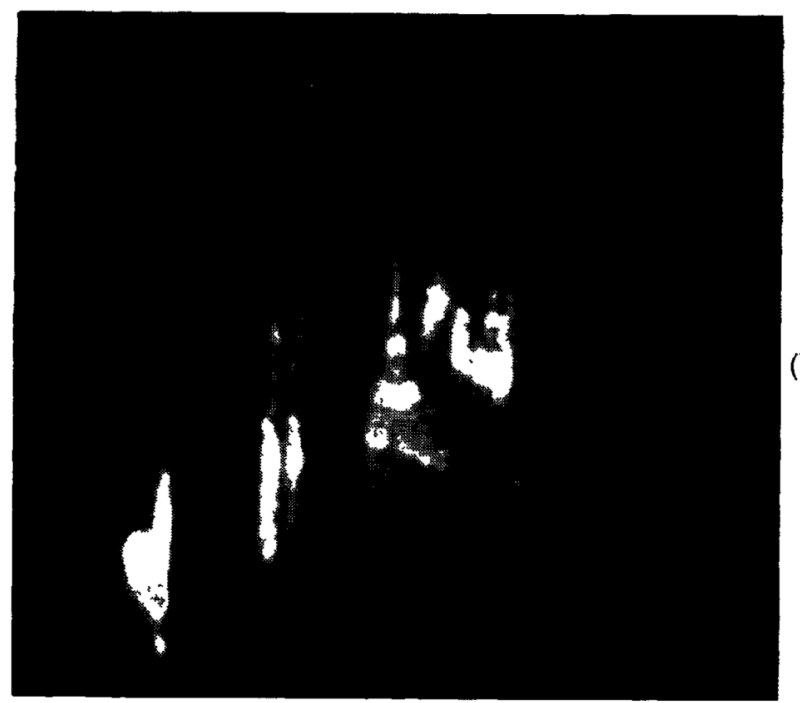

Photo. 8 Dark filed images of the hillock in Photo. 6 formed by the following reflections:
(a) $(111)_{\mathrm{M}}$
(b) $(002) z_{1}$

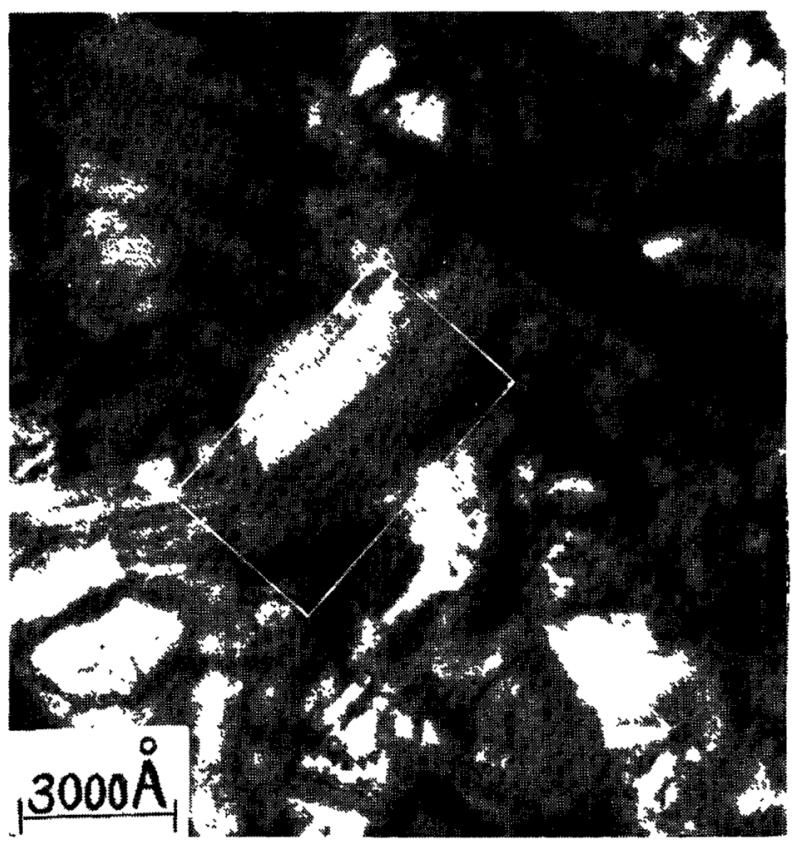

Photo. 9 Bright filed image of hillocks in the deposits with the (211) orientation

8-b which were taken from the same area of the hillock. A reversal of the contrast is clearly observed in these photographs. 


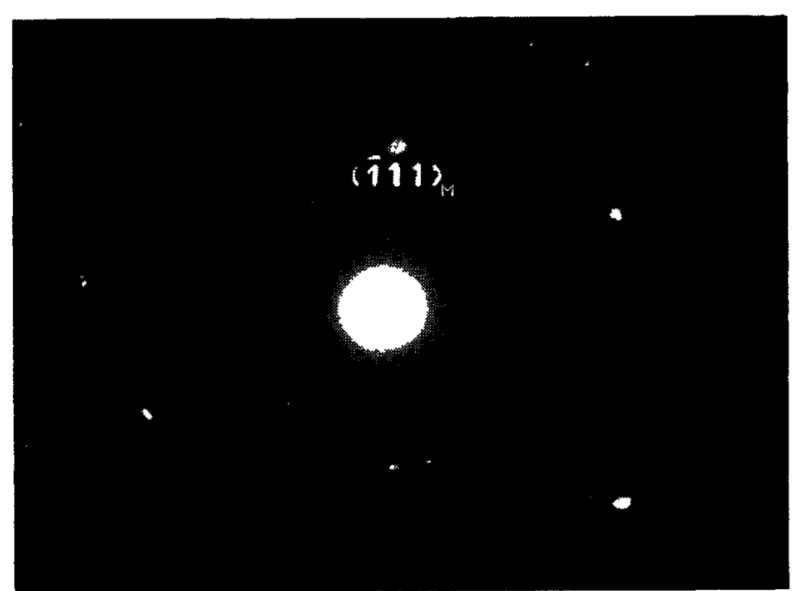

Photo. 10 Selected area electron diffraction pattern of the hillock surrounded by the rectangle in Photo. 9.

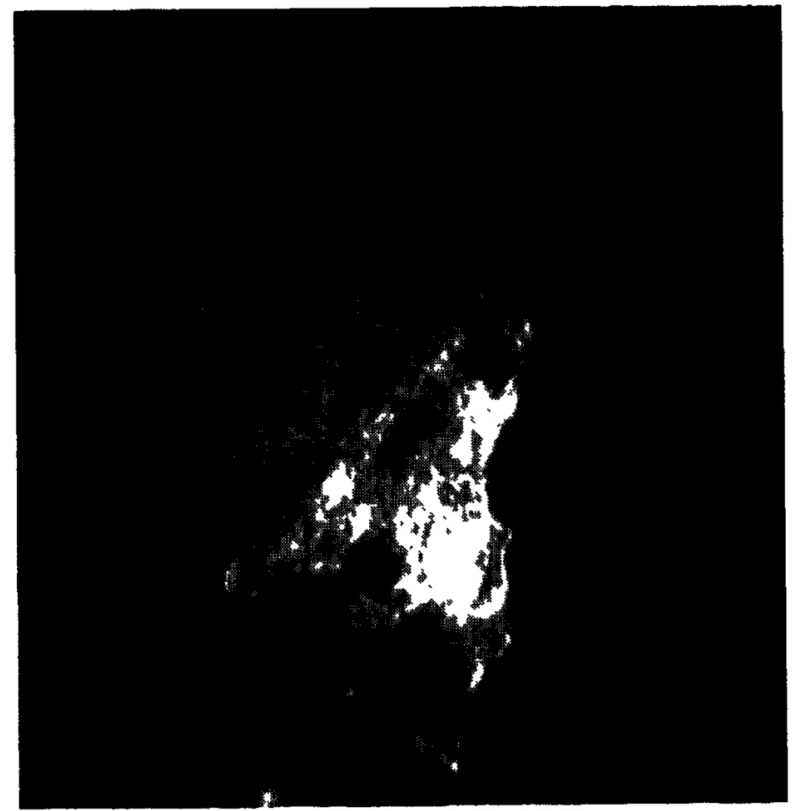

Photo. 11 Dark filed image of the hillock in Photo. 9 formed by the (111) $)_{M}$ reflection in Photo. 10

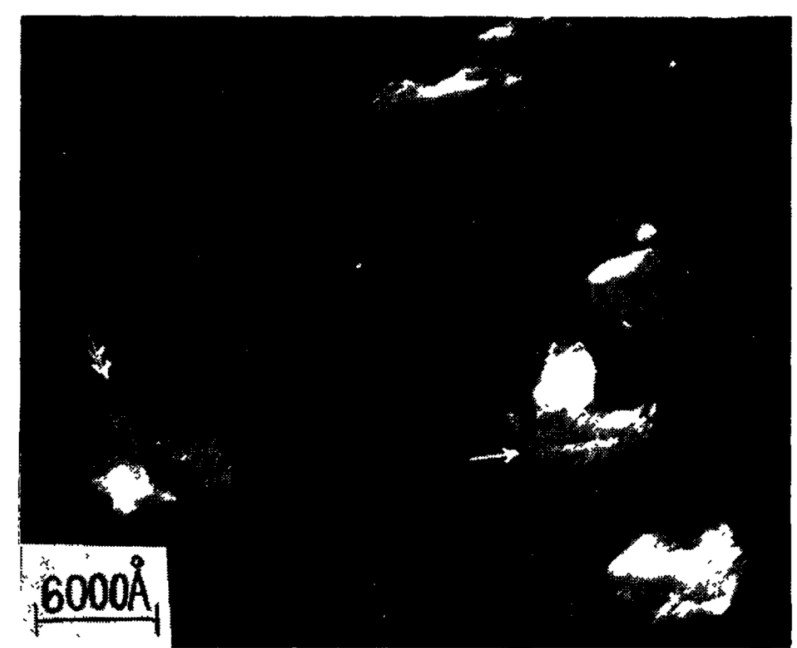

Photo. 12 Dark filod image of electrodeposited nickel with the (211) orientation formed by any (111) diffracted beam. Single and double arrows indicate twin boundaries of two kinds of hillocks.
A transmission electron micrograph of a hillock is shown in Photo. 9. An electron diffraction pattern obtained from the rectangular area in Photo. 9 is shown in Photo. 10. Photo. 11 shows a dark filed image formed by the $(\overline{1} 11)_{M}$ reflection in Photo. 10. In spite of the existence of streaks in Photo. 9, the hillok is wholly bright in Photo. 11 for the reason which will be mentioned later. A dark filed image of low magnification obtained by fixing the objective aperture at one position on the $\{111\}$ diffraction ring is shown in Photo. 12. A multitude of hillocks indicated by single arrows appear with almost the same orientation and a few hillocks marked by double arrows occur with another orientation. If the boundaries parallel to the arrow lines can be called center lines, the angle between the two center lines is about $110^{\circ}$.

\section{(2) Discussion of the surface structure}

The (110) reciprocal lattice plane of a crystal containing twins and the (211) reciprocal plane are given in Figs. 1 and 2. In Fig. 1, the points indicated by the subscript $M$ result from the crystal $M$. Crystals $Z_{1}$ and $Z_{2}$ which are twinned to the crystal $M$ on the $(\overline{1} 11)_{M}$ and $(1 \overline{1} 1)_{M}$ planes, respectively, give rise to the reciprocal lattice points indicated by the subscripts $Z_{1}$ and $Z_{2}$. If the reciprocal lattice points in Fig. 2 belong only to the crystal $M$, the points belonging to the crystal $Z_{1}$ twinned to the crystal M on the $(\overline{1} 11)_{M}$ plane are obtained by rotating Fig. 2 about the [111] axis by $180^{\circ}$, that is, by a symmetry operation by the plane perpendicular to the plane of the figure and containing $(02 \overline{2})$ and $(000)$ spots. The reciprocal lattice points of the crystal $Z_{1}$ obtained in

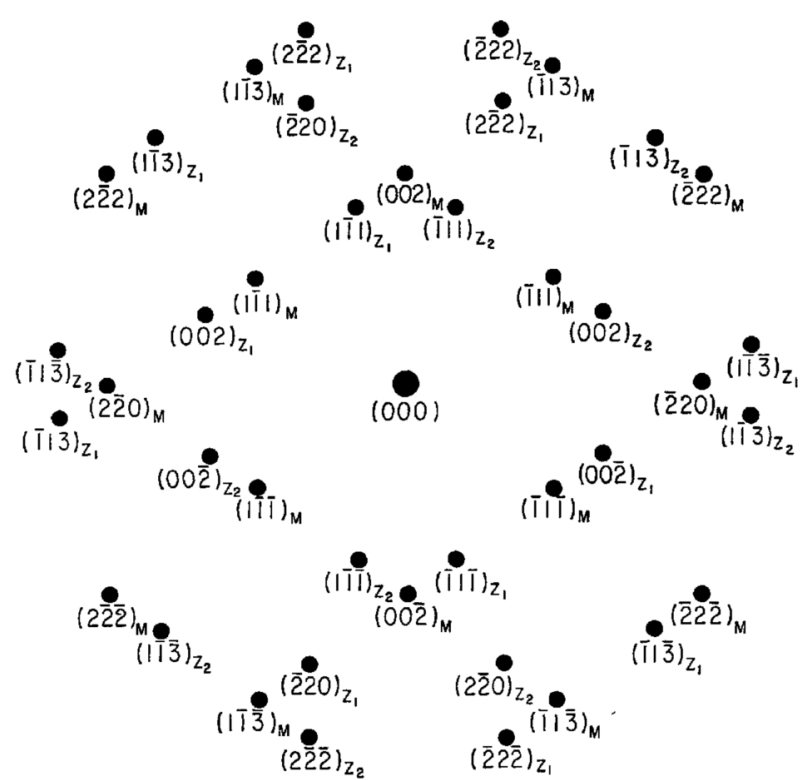

Fig. 1 The (110) reciprocal lattice plane of nickel.

$(\mathrm{hkl})_{\mathrm{M}}$ : Reciprocal lattice points of the crystal $\mathrm{M}$

$(\mathrm{hkl}) \mathrm{z}_{1}$ : Reciprocal lattice points of the crystal $\mathrm{Z}_{1}$ twinned to the crystal $\mathrm{M}$ on the (I11) M plane.

$(\mathrm{hkl}) \mathrm{Z}_{1}$ : Reciprocal lattice points of the crystal $\mathrm{Z}_{2}$ twinned to the crystal $\mathrm{M}$ on the $(\mathbf{I I 1})_{\mathrm{M}}$ plane 


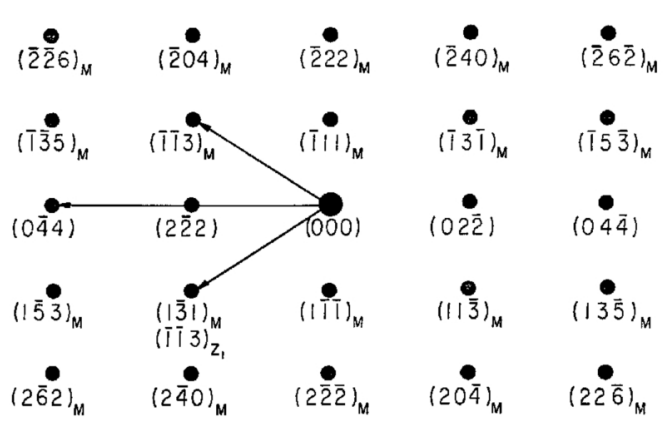

Fig. 2 The (211) reciprocal lattice plane of nickel. $(\mathrm{hkl})_{M}$ is the indices for the crystal $M$ and $(\mathrm{hkl}) \mathrm{Z}_{1}$ the indices for the crystal $Z_{1}$ twinned to the crystal $M$ on the (i11) $)_{M}$ plane

this way are superposed on those of the crystal M. Fig. 2 exhibits, therefore, the reciprocal lattice points on the (211) plane of the crystals $M$ and $Z_{1}$ which are twinned on the $(\overline{1} 11)_{M}$ plane perpendicular to the (211) plane.

Diffraction spots indexed in Photo. 7 correspond to the reciprocal lattice points of a crystal with the (110) orientation having the twin structure which is shown by the subscripts $M$ and $Z_{1}$ in Fig. 1 . The diffraction pattern of Photo. 7 was, therefore, obtained from the hillock with the (110) plane almost perpendicular to the incident electron beam, as a result of a local bending of the specimen film. The presence of diffraction spots indicated by the subscript $\mathrm{Z}_{1}$ shows the occurrence of the crystal $\mathrm{Z}_{1}$ twinned to the crystal $M$ on the $(\overline{1} 11)_{M}$ plane. On the other hand, from the absence of $\{200\}$ spots in Photo. 10 and the high diffracted intensity of the spot indicated by $(\overline{1} 11)_{\mathrm{M}}$, it is known that the diffraction pattern of Photo. 10 approximates to the point distribution of the (211) reciprocal lattice plane in Fig. 2, and that the (211) plane of the hillock as seen in Photo. 9 is almost perpendicular to the incident electron beam.

The reversal of bright and dark contrasts in Photos. 8-a and 8-b in which dark filed images are formed by the $(1 \overline{1} 1)_{M}$ and $(002) z_{1}$ reflections shows evidence that bright parts in Photo. 8-a correspond to the crystal M and those in Photo. 8-b to the crystal $Z_{1}$. The boundaries between bright and dark parts are the (111) $)_{M}$ twin boundaries. This is proved by the fact that no contrast appears in the dark filed image in Photo. 11 which is formed by the $(\overline{1} 11)_{M}$ reflection. The crystals $M$ and $Z_{1}$ are uniformly bright because of having the $(\overline{1} 11)_{M}$ plane as a common plane. It is, therefore, concluded that in the electrodeposited nickel with the (211) orientation prepared under the present electrodepositing condition, the crystals in Photo. 5 and the hillocks in Photo. 3 are twins composed of the crystals $M$ and $Z_{1}$ twinned to each other on the $(\overline{1} 11)_{M}$ plane perpendicular to the (211) plane, and hence, to the substrate surface. Thus, the surface of the deposits is covered by these twin hillocks.

If the film plane is exactly parallel to the (211) plane of each hillock, the $\{111\}$ ring in the transmission-electron diffraction pattern of Photo. 4 is composed of $(\overline{1} 11)_{M}$ spots from the hillocks. A dark filed image with low magnification in Photo. 12 is formed by a small part of the $\{111\}$ ring, and, therefore, only hillocks which have the same direction of twin boundaries should be observed bright on a whole. When some hillocks in this image shows such bright and dark contrasts as seen in Photo. 8-a, the [110] axis of the hillock is parallel to the incident electron beam and the $(\overline{1} 11)_{M}$ and $(1 \overline{1} 1)_{M}$ planes are perpendicular to the film plane due to the local bending of the film. The bright and dark contrasts are known to result from the $(1 \overline{1} 1)_{\mathrm{M}}$ reflection. The $(1 \overline{1} 1)_{\mathrm{M}}$ spot in Fig. 1 and the (111) $)_{M}$ spot in Fig. 2 are superposed on each other in the electron diffraction pattern of a limited field in which the two orientations of hillocks mentioned above are observed. Therefore, in the dark filed image formed by the superposed $\{111\}$ reflection, the angle between the (i11) ${ }_{M}$ twin boundaries of both kinds of hillocks being brightened by the $(\overline{\mathbf{1}} 11)_{\mathrm{M}}$ and $(111)_{M}$ reflections should be $109^{\circ} 37^{\prime}$, corresponding to the angle made by $(1 \overline{1} 1)_{M}$ and $(\overline{1} 11)_{M}$ spots at (000) in Fig. 1. The angle is in good agreement with the value of about $110^{\circ}$ measured in Photo. 12 , and this strongly supports the conclusion mentioned in the last paragraph.

Double diffraction can occur from the $(1 \overline{1} 1)_{\mathrm{M}}$ or $(11 \overline{1})_{M}$ plane of the crystal $M$ and the $(11 \overline{1}) z_{1}$ or $(111) z_{1}$ plane of the crystal $Z_{1}$, when the surface of the electrodeposited nickel having many twin hillocks consisting of crystals $M$ and $Z_{1}$ twinned to each other on the (111) $)_{M}$ plane perpendicular to the (211) plane in parallel with the substrate surface is examined by reflection electron diffraction ${ }^{(6)}$. On the other hand, these (1i1) and (11i) planes are not parallel to the electron beam in transmission electron diffraction when the (211) plane is parallel to the surface. Therefore, no diffraction arises from these $\{111\}$ planes as shown in Fig. 2 and double diffraction cannot occur from these planes. When the electron beam is perpendicular to the (211) plane, double diffraction can result from the crystals $M$ and $Z_{1}$ twinned to each other on the $(\overline{1} 11)_{M}$ plane, but all resulting spots are superposed on primarily diffracted spots and new spots are not formed. For exmaple, if a diffracted beam

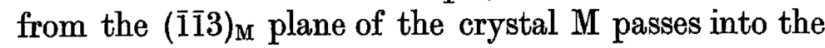
crystal $Z_{1}$ and suffers a secondary diffraction from the $(\overline{1} \overline{1} 3)_{z_{1}}$ plane as indicated by arrows in Fig. 2, a double diffraction spot appears at the $(0 \overline{4} 4)$ position. Consequently, no double diffraction rings are observed in the transmission electron diffraction pattern of Photo. 4.

Diffraction rings due to a close-packed hexagonal phase are not observed at all in Photo. 4. It has already been reported by the author ${ }^{(6)}$ that the double diffraction spot appears at the position corresponding to the $(10 \overline{1} 0)$ reflection of a close-packed hexagonal phase with the $(1010)$ orientation in the reflection electron diffraction pattern. Therefore, the existence of close-packed hexagonal phase is considered unnecessary and impossible for the electrodeposited nickel with the (211) orientation. Wright and Goddard (9) recently reported parallel growth of the hexagonal phase in nickel electrodeposited on the (110) plane of copper single crystals and on the (100)

(9) J.G. Wright and J. Goddard : Phil. Mag., 11 (1965), 485. 
plane of $a$-cobalt single crystals. Growth of the hexagonal phase may occur during electrodeposition of nickel on the single crystal substrates having nearly the same lattice parameters as that of the hexagonal nickel. In the present study, however, the closepacked hexagonal phase has not been observed to grow during electrodeposition of nickel on the mechanically polished substrates.

\section{The surface structure and chemical reac- tivity}

As shown in Photo. 5, the area in the vicinity of gxain boundaries are dissolved during dissolution of the electrodeposited copper of the middle layer. Photo. 13 shows an electron micrograph of a specimen corroded in a $5 \% \mathrm{FeCl}_{3}-\mathrm{CH}_{3} \mathrm{OH}$ solution for $2 \mathrm{sec}$ before dissolution of the electrodeposited copper of the middle layer. Coherent twin boundaries appear bright in Photo. 13. This is caused by a selective dissolution, but there may be the possibility that the brightness arises from a diffraction contrast. As mentioned above, there are many locally bending parts in the film. If this possibility were valid, the contrast would change at the bending parts. In effect such a change is not observed at all, and the brightness is caused not by any diffraction contrast but by dissolution in the corrosive solution in which the pitting corrosion is well known to occur easily. The author ${ }^{(1),(10)}$ has already elucidated the cause of the chemical reactivity of twin boundaries. A large number of twin boundaries are observed on the surface of the electrodeposited nickel with the (211) orientation, and as these boundaries are chemically reactive, the electrodeposited nickel having this orientation will be chemically less stable in corrosive environment.

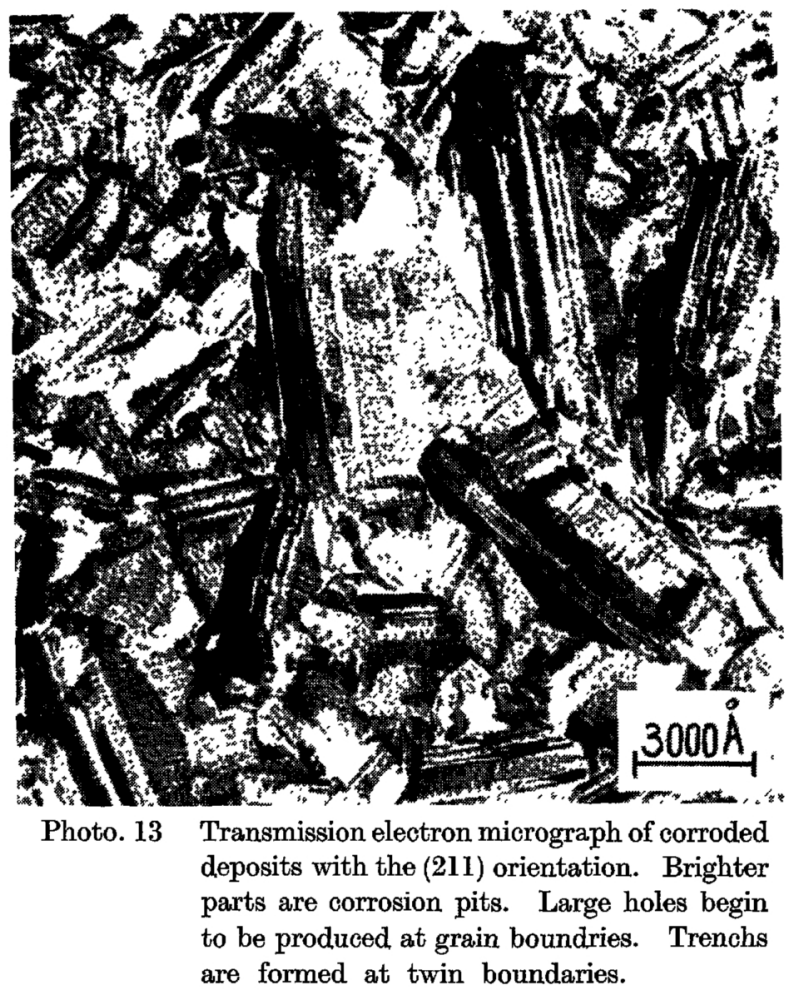

(10) K. Hashimoto : Corrosion Science, 5 (1965), 597.
In the electrodeposited nickel with the (210) orientation stacking faults and twin boundaries are initially dissolved in a corrosive solution, and corrosion holes appear. Twin spots of crystals twinned on $\{111\}$ planes are observed in the reflection electron diffraction pattern of the 2nd type electrodeposits. However, twin boundaries and stacking faults are observed not to appear frequently in the transmission electron micrographs of the electrodeposited nickel with the (210) orientation, and a few corrosion holes are observed in the specimens corroded by immersion in a corrosive solution for the same period of time as is the case for the (211) orientation specimen before dissolution of the deposited copper of the middle layer. It is, therefore, probable that the electrodeposited nickel with the (210) orientation is chemically more stable than that with the (211) orientation. It is also possible that the densities of chemically reactive crystal defects such as twin boundaries and stacking faults are low on the surface of the electrodeposited nickel with the (210) orientation. If this is valid and the densities of the crystal defects differ depending upon orientations of the deposits, it is very interesting fact to be confirmed from the viewpoint of crystal growth.

In the reflection electron diffraction pattern of the electrodeposited nickel with the (110) orientation ${ }^{(6)}$, twin spots do not appear. The twinning on the two $\{111\}$ plane perpendicular to the (110) plane might be possible but cannot be confirmed, because this twinning does not produce any reflections other than those from the matrix. The surface of the deposits with this orientation appears very flat in electron micrographs using replica and does not show the existence of any crystals twinned on the $\{111\}$ planes perpendicular to the (110) plane as observed in the deposits with the (211) orientation(6). Growth of such twinned crystals is also seldom observed in transmission electron micrographs. Corrosion holes occur at grain boundaries by immersion in a corrosive solution, but in small quantities.

It seems therefore that the electrodeposited nickel with the $(210)$ or $(110)$ orientation prepared under the present electrodepositing conditions is more chemically stable than the electrodeposited nickel with the (211) orientation.

\section{Conclusion}

The surface structures of electrodeposited nickel with (211), (210) and (110) orientations have been investigated by means of transmission electron microscopy. With increasing thickness of the nickel electrodeposited on the mechanically polished copper disks, the characteristic preferred orientation appears depending on the electrodepositing condition. Special procedures have been devised in order to directly observe the structure of the thin top layer of thick nickel deposits having the preferred orientation by transmission electron microscopy.

A large number of hillocks twinned on the (1111) plane perpendicular to the (211) plane are observed in the deposits with the (211) orientation. Twin 
and grain boundaries are chemically reactive and initially dissolved in a corrosive solution. In the electrodeposited nickel having the (210) or (110) orientation, the dissolution begins to occur at the stacking faults and twin boundaries in a corrosive solution.

It is probable that the electrodeposited nickel with the (211) orientation is more chemically reactive than that having the other two orientations, when prepared under the present electrodepositing conditions. Transmission electron micrography is profitable to investigate the relationship between the surface structure and chemical reactivity of electrodeposited metals.

\section{Acknowledgements}

The author wishes to express his deep appreciation to Professor Saburo Shimodaira and Professor Shiro Ogawa of the Research Institute for Iron, Steel and Other Metals, Tohoku University, Sendai, Japan, for their continuous encouragement and advice. 